\title{
Características biomecânicas, ergonômicas e clínicas da postura sentada: uma revisão
}

\author{
Biomechanic, ergonomic, and clinical features of the sitting posture: a review
}

\author{
Nise Ribeiro Marques, Camilla Zamfolini Hallal', Mauro Gonçalves ${ }^{2}$
}

Estudo desenvolvido no Laboratório de Biomecânica do Depto. de Educação Física da Unesp - Universidade Estadual Paulista, campus de Rio Claro, RioClaro, SP, Brasil

1 Fisioterapeutas; mestrandas no PPG-Ft - Programa de PósGraduação em Fisioterapia, Depto. de Fisioterapia da Unesp - campus de Presidente Prudente, SP

2 Fisioterapeuta; Prof. Dr. do PPG-Ft, Depto. de Educação Física da Unesp Rio Claro

\section{ENDEREÇO PARA CORRESPONDÊNCIA}

Nise R. Marques

Depto. de Educação Física/ Unesp

Av. 24-A n. 1515

13506-900 Rio Claro SP e-mail:

nisermarques@yahoo.com.br

\section{APRESENTAÇÃO}

nov. 2009

ACEITO PARA PUBLICAÇÃO abr. 2010
Resumo: A posição sentada é a mais adotada nos ambientes de trabalho, na escola e nas atividades de lazer. Porém, a manutenção prolongada dessa posição ocasiona a adoção de posturas inadequadas e sobrecarrega as estruturas do sistema musculoesquelético, o que pode acarretar dor e lesão na coluna lombar. A presente revisão teve como objetivo identificar os fatores biomecânicos, ergonômicos e clínicos envolvidos na sustentação da postura sentada. Para isso, foram consultadas as bases de dados ISI Web of Knowledge, Medline, Pubmed e EBSCO Host, sendo selecionados 72 artigos publicados entre 1965 e 2010 . Foi possível identificar que na posição sentada não existe uma postura ideal a ser sustentada, mas algumas posturas são mais recomendadas do que outras, tal como a postura sentada ereta e a postura lordótica. As cadeiras influenciam o padrão da posição sentada: conforme seu design, pode permitir maior variedade de posturas. Modificações na cadeira e a utilização de exercícios para o aumento da resistência muscular e da propriocepção, bem como a reeducação postural, são intervenções úteis para reduzir o impacto causado pela posição sentada prolongada no sistema musculoesquelético.

DescritoRes: Biomecânica; Dor lombar; Engenharia humana; Fisioterapia (Especialidade); Postura

ABSTRACT: The sitting posture is the most adopted in work environment, at school and in leisure activities. However, prolonged maintenance of this position causes the adoption of inadequate postures and overloads skeletal muscle structures, which may lead to spine pain and injuries. The purpose of the present review was to identify biomechanic, ergonomic, and clinical features involved in maintenance of the sitting position. The search in ISI Web of Knowledge, Medline, Pubmed and EBSCOHost data bases led to selecting 72 articles published between 1965 and 2010. Results show that there is not an ideal posture to be kept, but some postures are more recommended than others, like the upright sitting and the lordotic postures. Chairs influence the pattern of sitting, and their design allows for posture diversity. Changes in the chair, exercises to improve endurance and proprioception, as well as postural re-education, are useful interventions to reduce the impact of prolonged sitting position on the skeletal muscle system.

Key words: Biomechanics; Human engineering; Low back pain; Physical therapy (Specialty); Posture 


\section{INTRODUÇÃO}

O rápido desenvolvimento tecnológico nos países industrializados, a automação e a informatização dos postos de trabalho, que ocorreu a partir da segunda metade do século $X X$, foram grandes responsáveis pela adoção cada vez mais freqüente da posição sentada nos postos de trabalho ${ }^{1-3}$.

Quando mantida por longos períodos, a posição sentada leva a prolongada sustentação da flexão lombar, redução da lordose nessa região e sobrecarga estática nos tecidos osteomioarticulares da coluna, fatores esses que estão diretamente relacionados ao desenvolvimento da dor lombar ${ }^{4-7}$. A relação entre a posição sentada e a dor lombar estimulou o desenvolvimento de diferentes tipos de cadeiras para uma melhor adequação ergonômica. Alguns pesquisadores introduziram a idéia da utilização de cadeiras mais flexíveis e de bolas de exercícios, que permitiriam maior movimentação e auxiliariam na redução da sobrecarga estática na coluna vertebral ${ }^{8-10}$. Outros pesquisadores preferiram, no entanto, investir na modernização e adequação de assentos, encostos e suportes, que proveriam melhor estabilidade à coluna e diminuiriam a ocorrência de fadiga muscular ${ }^{11-14}$.

Entretanto, apesar do desenvolvimento da ergonomia no que tange à confecção de cadeiras e móveis utilizados nos postos de trabalho, esses instrumentos foram pouco eficazes para a prevenção de dores lombares. Assim, atualmente, o principal foco para a redução da ocorrência desse sintoma musculoesquelético é a introdução da prática de exercícios e a adoção de programas de reeducação postural ${ }^{15}$.

A presente revisão teve como objetivo identificar os fatores biomecânicos, ergonômicos e clínicos envolvidos na sustentação da postura sentada.

METODOLOGIA

Foi realizada busca de estudos científicos em inglês nas bases de dados ISI Web of Knowledge, Medline, Pubmed e EBSCOHost utilizando as palavras-chave: seat, electromyography and seat, intradiscal pressure and seat, prolonged sitting, anthropometry and sitting posture, anthropometry and chair, sitting posture and biomechanics, sitting posture and chair, sitting posture and EMG, sitting posture and low back pain, sitting posture and human engineering e sitting posture and backache. Foram encontrados 72 artigos publicados entre 1965 e 2010, dos quais a maior parte, os mais significativos, são comentados e discutidos a seguir.

\section{A POSTURA SENTADA}

A Academia Americana de Ortopedia define postura como o estado de equilíbrio entre músculos e ossos com capacidade para proteger as demais estruturas do corpo humano de traumatismos, seja na posição em pé, sentado ou deitado. A postura também pode indicar a posição relativa dos segmentos corporais durante o repouso ou atividade. Assim, a manutenção de uma boa postura durante uma atividade específica depende de uma interação complexa entre as funções biomecânicas e neuromusculares ${ }^{16}$.

Uma boa postura deve ser aquela que previne movimentos compensatórios, distribui adequadamente as cargas e conserva energia. Entretanto, apesar da grande aceitação clínica dos aspectos que influenciam a formação de uma boa postura, pouco se sabe acerca de métodos quantitativos para caracterizá-la ${ }^{17}$.

A posição sentada, por sua vez, é definida como a situação na qual o peso corpóreo é transferido para o assento da cadeira por meio da tuberosidade isquiática, dos tecidos moles da região glútea e da coxa, bem como para o solo por meio dos pés ${ }^{15}$. No entanto, sentar é uma ação dinâmica que deve ser vista como um comportamento e não somente como uma condição estática ${ }^{18}$.

A posição sentada é estudada há vários séculos e os conceitos acerca de uma postura ideal nessa posição se alteraram ao longo de décadas. No século XIX a crença na necessidade de sentar-se com as costas retas refletiu-se no design do mobiliário escolar da época, que apresentava encostos verticais e assentos horizontais planos. No século XX, o foco na adequação ergonômica das cadeiras aumentou, sendo desenvolvidos assentos e encostos com formatos que se adéquam melhor ao corpo humano. As cadeiras passaram a possuir molas e amortecedores para aumentar a possibilidade de movimentação e o conforto ${ }^{11}$.

Para entender melhor a posição sentada, vários pesquisadores classificaram essa posição de acordo com diferentes pontos de referência. Harrison et al. ${ }^{19}$ utilizou o posicionamento do centro de massa (CM) e classificou a posição sentada em três diferentes posturas: a anterior, caracterizada pelo posicionamento do CM à frente da tuberosidade isquiática e obtida quando a pelve é antevertida; a posterior, caracterizada pela posição posteriorizada do CM em relação à tuberosidade isquiática e obtida com o movimento de retroversão da pelve; e a medial, que é caracterizada pelo posicionamento do CM coincidente com a tuberosidade isquiática e ocorre quando a coluna lombar está em posição neutra.

Pynt et al. ${ }^{15}$ classificaram a posição sentada de acordo com as curvaturas da coluna em: postura sentada flexionada, que ocorre quando há inversão da curvatura normal da coluna lombar, o que gera uma cifose nessa região; e postura lordótica, que ocorre com a manutenção da curvatura lombar normal. O'Sullivan et al. ${ }^{20}$ e Callaghan e Dunk ${ }^{21}$ também classificaram a posição sentada conforme as curvaturas da coluna em: postura lombo-pélvica sentada ereta, definida como a postura na qual a pelve, a lordose lombar e a cifose torácica estão em posição neutra (Figura 1A); em postura sentada em relaxamento (slump), caracterizada pela retroversão da pelve, o que reduz a lordose lombar (Figura 1B); e em postura torácica ereta, com a anteversão da pelve, que gera um aumento da curvatura lombar (Figura 1C).

Além da postura, outro aspecto importante ao analisar a posição sentada é o tempo em que esta é sustentada ${ }^{22}$. Várias mudanças na postura são recomendáveis para não gerar desconforto ou fadiga e o tempo médio de intervalo entre duas trocas consecutivas deveria ser de $5 \mathrm{mi}$ nutos $^{23}$. Além disso, a permanência nessa posição por mais de quatro horas re- 

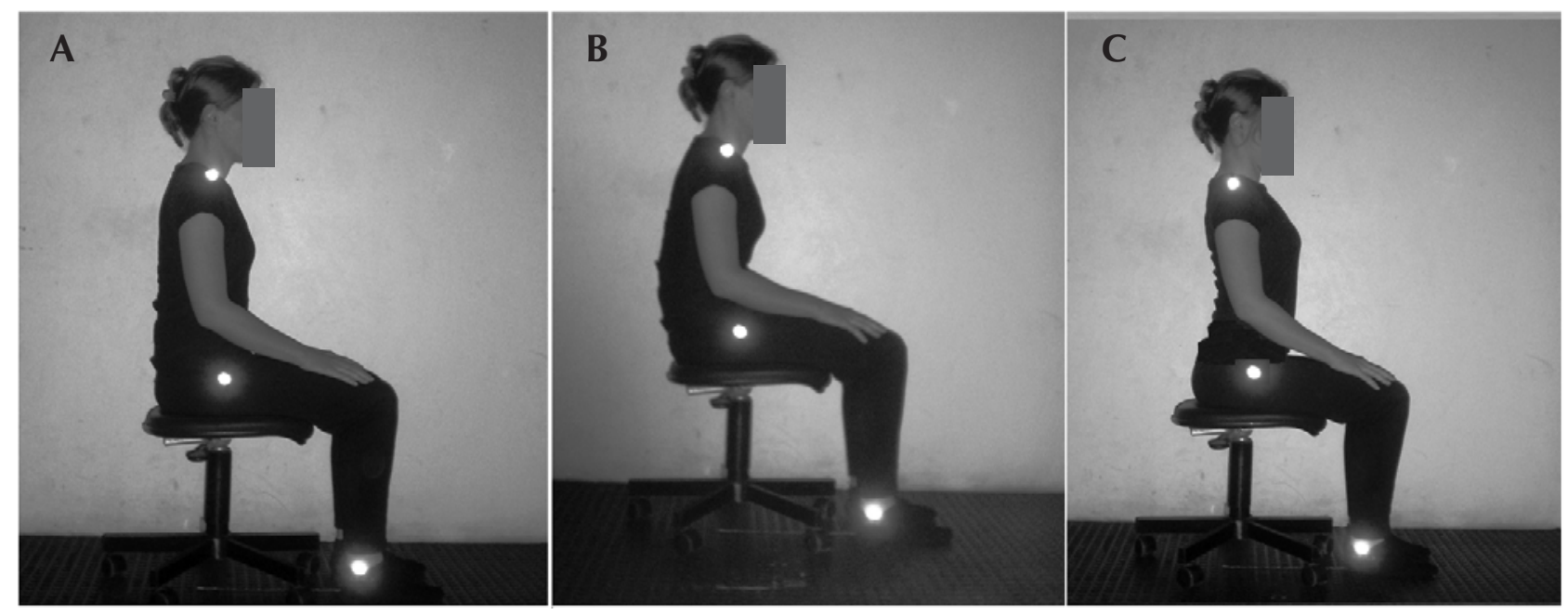

Figura 1 (A) Postura sentada lombo-pélvica ereta ou lordótica, com pelve e coluna lombar em posição neutra; (B) postura sentada relaxada (slump), com retroversão pélvica e redução da curvatura lombar; (C) postura torácica ereta, com anteversão pélvica e aumento da curvatura da coluna lombar ${ }^{15,21,22}$

presenta um risco para o desenvolvimento de dor lombar ${ }^{24}$

\section{Variáveis biomecânicas}

O posicionamento das curvaturas da coluna está relacionado com a distribuição das cargas. A postura sentada reta, na qual os ângulos dos quadris, tronco, joelhos e tornozelos são mantidos em $90^{\circ}$, cria tensão nos isquiotibiais e nos glúteos, o que causa retroversão da pelve, horizontaliza o ângulo sacral e retifica a lordose lombar. Isso gera um aumento das cargas compressivas no disco intervertebral, além de acarretar fadiga dos eretores espinhais (músculos que devem estar ativos para manter a postura sentada ereta) ${ }^{15}$.

A postura sentada cifótica da coluna lombar, caracterizada quando o ângulo entre as vértebras S1 e L1 é menor ou igual a $22^{\circ}$, aumenta em $85 \%$ a pressão intradiscal (PID). A carga compressiva e a manutenção por mais de seis horas na postura cifótica reduz a altura do disco em 2,1 mm $\mathrm{mm}^{25,26}$. Essa redução na altura do disco intervertebral pode acarretar degeneração discal ${ }^{27,28}$.

A postura sentada lordótica, caracterizada pela manutenção de um ângulo de aproximadamente $47^{\circ}$ de lordose lombar, apresenta redução da PID, quando comparada à postura cifótica. Essa redução na PID é resultado da baixa tensão nos ligamentos posteriores e redução da atividade dos músculos extensores da coluna $^{29}$. No entanto, estudos recentes mostraram que esta apresenta valores semelhantes na posição em pé e sentada. Isso está relacionado com o tipo de método utilizado na mensuração da $\mathrm{PID}^{30}$. Na década de 1960, os valores encontrados por Nachemsom ${ }^{30}$, que utilizou transdutor líquido de polietileno, foram de 1,24 MPa na posição sentada, enquanto Wilke et al. ${ }^{31}$, no final da década de 1990, que utilizaram transdutores piezoresistivos, encontraram valores de 0,5 MPa para a postura em pé e 0,6 MPa para a postura sentada. Além disso, um estudo que mediu a PID por meio de transdutores de agulha identificou uma ampla variação nos valores médios entre os sujeitos, além de verificar valores menores de PID na posição sentada em relação à posição em pé32. Assim, se a posição sentada é um fator causal da dor lombar, isso não pode ser diretamente relacionado aos efeitos dessa posição na $\mathrm{PID}^{32}$.

Estudos eletromiográficos também demonstraram que as posturas com aumento ou redução na curvatura lombar, quando comparadas a postura com essa curvatura em posição neutra, apresentam menor atividade dos músculos oblíquo interno e multífidos lombares superficiais ${ }^{33,34}$. Além disso, a manutenção da postura neutra também auxilia o controle neuromuscular dessa região ${ }^{17}$.

\section{Aspectos ergonômicos}

Na posição sentada, a maior parte do peso do corpo é transferida para uma área de suporte na tuberosidade isquiática e tecidos moles. Assim, se não houver um apoio correto na região lombar, a pressão intradiscal pode ser elevada em até $35 \% \%^{35,36}$. Para reduzir a pressão intradiscal é necessária a inclinação para trás do encosto e a presença de suporte lombar adequado ${ }^{37,38}$.

Os suportes lombares, apoios de braços, inclinação do assento e do encosto, a liberdade para movimentação, as cadeiras com regulagem de altura e o assento curvado anteriormente têm sido apontados como componentes ergonômicos redutores da sobrecarga no sistema musculoesquelético na posição sentada e estão associados à elevação das taxas de conforto ${ }^{39,40}$.

Os suportes lombares de até $3 \mathrm{~cm}$ promovem maior apoio para essa região e previnem a diminuição da curvatura lombar. Nesse sentido, Goossens et al. ${ }^{41}$ utilizaram eletromiografia para verificar a influência do suporte escapular no efeito do suporte lombar. Para isso utilizaram cinco diferentes distâncias $(0,2,4,6$ e $8 \mathrm{~cm}$ ) entre a tangente do apoio lombar e a tangente paralela do apoio escapular (Figura 2), sendo essas distâncias chamadas de espaço livre para o ombro. Os autores concluíram que o espaço livre para o ombro deve ser de pelo menos 6 $\mathrm{cm}$ para permitir melhor adaptação ao contorno da coluna e fornecer apoio lombar $^{41}$. A Figura 2 representa os efeitos do suporte lombar na maior distância (A), na intermediária (B) e na menor distância 

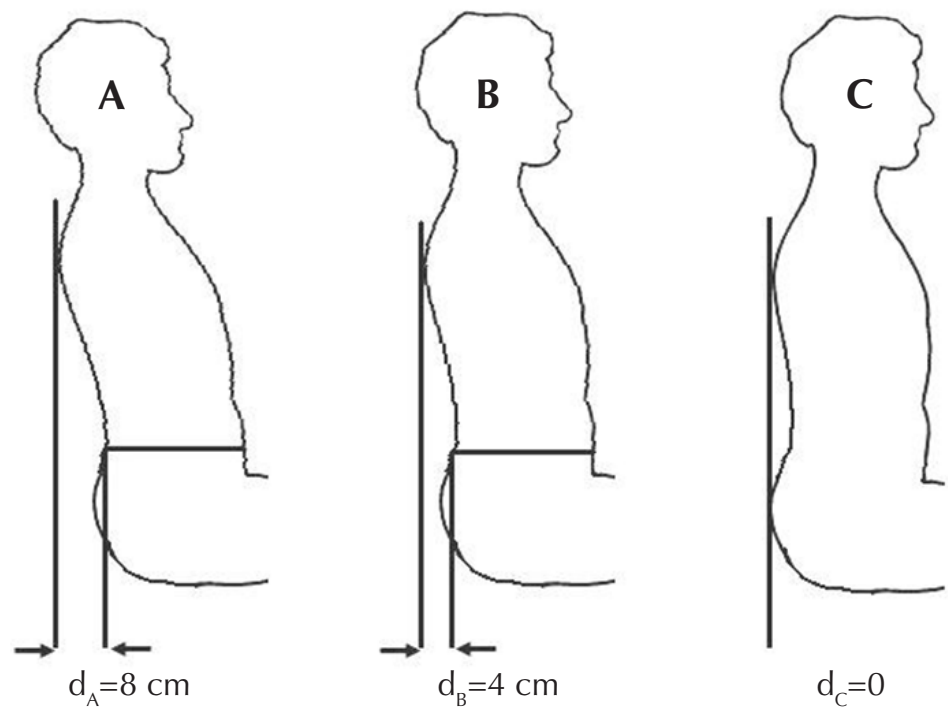

Figura 2 Diferentes distâncias de espaço livre para ombro na postura sentada ${ }^{41}$

(C) de espaço livre para os ombros.

Resultados de estudos sobre a atividade eletromiográfica e das pressões intradiscais revelaram que o ângulo de inclinação assento/encosto deve ser de aproximadamente $120^{\circ}$ horizontalmente, considerando a região posterior do assento no máximo $10^{\circ}$ inclinada para trás. A altura correta do assento deve ser menor que a distância do joelho ao pé, o que elimina a pressão na fossa poplítea ${ }^{42}$. Os encostos devem estar um pouco abaixo dos ombros (pelo menos $6 \mathrm{~cm}$ ) para evitar que ocorra extensão na coluna lombar com um conseqüente aumento na lordose dessa região, gerando assim anteriorização da vértebra L5 (Figura 3) ${ }^{42}$.

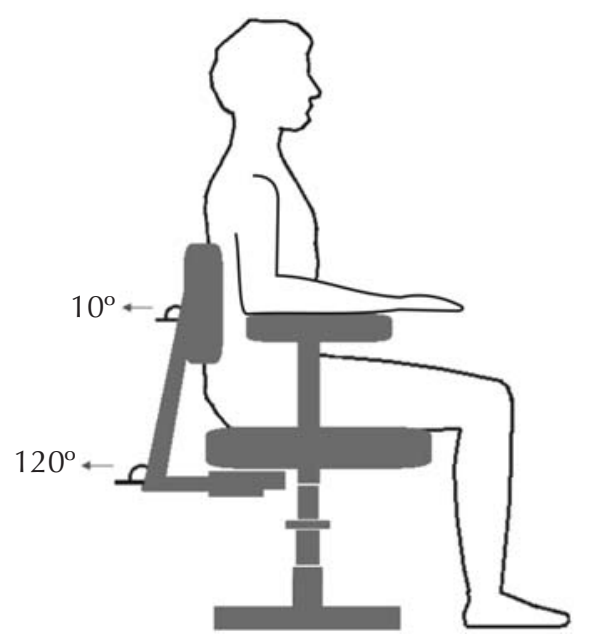

Figura 3 Adaptações ergonômicas na cadeira de escritório ${ }^{42}$
As cadeiras dinâmicas, que têm molas e amortecedores, aumentam a possibilidade de movimentação do indivíduo na posição sentada. Isso contribui para a difusão dos nutrientes através da placa vertebral terminal, o que promove a nutrição do disco intervertebral ${ }^{9,10,43}$. O uso de bolas de exercícios em comparação ao uso de cadeiras de escritório convencionais não apresenta diferenças significativas na atividade eletromiográfica dos músculos do tronco ${ }^{10,44}$. Entretanto, sentar-se em uma bola de exercícios gera coativação dos músculos flexores e extensores de quadril, o que possibilita maior estabilidade da coluna lombar e melhor controle neuromuscular ${ }^{44}$.

A antropometria também é outro fator que deve ser levado em consideração. Vários estudos recentes sugerem que existe uma incompatibilidade entre as medidas antropométricas e o mobiliário encontrado em escolas e postos de trabalho. Nesse sentido, são recomendadas a utilização de mesas e cadeiras equipadas com mecanismos ajustáveis de altura e largura ${ }^{45-47}$.

\section{Efeitos no sistema musculoesquelético}

A estabilidade da região lombopélvica ocorre pela ação interdependente de três subsistemas: o passivo, composto pelas estruturas articulares da coluna; o ativo, composto pelos músculos do tronco; e o neural, formado pelas estruturas do sistema nervoso. Assim, quando algum desses subsistemas apresenta falha, os demais operam de modo compensatório para manter a estabilida$\mathrm{de}^{48}$. No entanto, se essas compensações ultrapassarem os limiares fisiológicos das estruturas, gerando sobrecarga mecânica, ocorrerá lesão ${ }^{48}$.

A adoção de posturas inadequadas na posição sentada altera a atividade muscular e desencadeia mecanismos que põem em risco a integridade do sistema musculoesquelético. Snidjers et al. ${ }^{49}$ documentaram uma redução na atividade eletromiográfica do músculo oblíquo interno quando compararam a posição sentada ereta à posição sentada com as pernas cruzadas, sugerindo que isso reflete o maior recrutamento das estruturas passivas para estabilizar a articulação sacroilíaca ${ }^{49}$.

O funcionamento do subsistema neural, composto por mecanorreceptores e nervos, também é afetado pela sobrecarga das estruturas passivas. Segundo Gedalia et al..$^{50}$, quando as estruturas viscoelásticas se apresentam sobrecarregadas e deformadas, a sensibilidade dos mecanorreceptores diminui e há perda na resposta reflexa dos multífidos, comprometendo a estabilidade e propriocepção local.

Para tentar reduzir o impacto da postura sentada nas estruturas osteomioarticulares, algumas posturas são apontadas como mais saudáveis, como a postura lordótica, que diminui a pressão intradiscal, a degeneração do disco e exibe menores níveis de lesão por tensão ligamentar ${ }^{15}$. Além disso, movimentos como estender as pernas e colocar um tornozelo sobre o outro contribuem para prover estabilidade à pelve e coluna lombar ${ }^{19}$.

No entanto, apenas a adoção de uma boa postura e a utilização de mobiliário correto não são medidas suficientes para reduzir a sobrecarga nos tecidos osteomioarticulares. Um bom condicionamento do sistema muscular também é necessário para prover estabilidade à coluna $^{15}$. Nesse sentido, Pelham et al. ${ }^{51}$ e Womersley et al. ${ }^{52}$ sugeriram que no tratamento da dor lombar de origem postural as condutas mais indicadas seriam a interrupção de longos períodos na posição sentada, adequação do mo- 
biliário e a realização de exercícios e técnicas manipulativas, como por exemplo o método McKenzie.

Além da dor lombar, outros sintomas e doenças musculoesqueléticas estão presentes em indivíduos que mantêm a postura sentada por longo tempo. Dores no pescoço, costas, joelhos e coxas são comuns entre os motoristas de ônibus (entre 35 e 60\%) com jornada de trabalho de 9 a $10 \mathrm{~h}$ por dia durante cinco dias na semana ${ }^{53}$.

Portanto, intervenções com exercícios que aprimorem a resistência muscular e a propriocepção, bem como a reeducação postural, são atualmente utilizadas para diminuir os efeitos negativos da posição sentada prolongada ${ }^{15}$.
CONCLUSÃO

O sentar é uma situação dinâmica que deve ser vista como um comportamento e não somente como uma condição estática. Assim, na posição sentada não existe uma determinada postura a ser sustentada. Entretanto, algumas posturas são mais recomendadas do que outras, tais como a postura sentada ereta e a postura lordótica. Além disso, um tempo de manutenção da postura sentada maior que quatro horas pode representar um risco para o sistema musculoesquelético.

As cadeiras também influenciam o comportamento de sentar, sendo que algumas permitem maior troca de posturas. Acessórios como suportes lomba- res e apoios para braços, bem como a inclinação e a altura do encosto e do assento são componentes ergonômicos que reduzem a carga mecânica na coluna durante a posição sentada.

Posturas inadequadas, fadiga muscular, baixa propriocepção e sobrecarga nas estruturas osteomioarticulares causadas pela posição sentada prolongada são fatores de risco para o aparecimento de dor e lesão lombar. Dessa forma, um conjunto de medidas como modificações no mobiliário, exercícios para o aumento da resistência muscular e propriocepção, bem como a reeducação postural são intervenções importantes para reduzir o impacto do sentar no sistema musculoesquelético.

\section{REFERÊNCIAS}

1 Graf M, Guggenbühl U, Krueger H. An assessment of seated activity and postures at five workplaces. Int J Ind Ergon. 1995;15(2):81-90.

2 Li G, Haslegrave CM. Seated work postures for manual, visual and combined tasks. Ergonomics. 1999;42(8):1060-86.

3 Hartivigsen J, Lebouf-Yde C, Lings S, Corder EH. Is sitting-while-at-work associated with low-back pain? A systematic, critical literature review. Scand J Public Health. 2000;28:230-9.

4 Black KM, McClure P, Polansky M. The influence of different sitting positions on cervical and lumbar posture. Spine. 1996;21(1):65-70.

5 Callaghan JP, McGill SM. Low back joint loading and kinematics during standing and unsupported sitting. Ergonomics. 2001;44(3):280-94.

6 Adams MA, Dolan P. Recent advances in lumbar spinal mechanics and their clinical significance. Clin Biomech (Bristol, Avon). 1995;10(1):3-19.

7 Makhous M, Lin F, Hendrix RW, Hepller M, Zang LQ. Sitting with adjustable ischial and back supports: biomechanical changes. Spine. 2003;28(11):1113-22.

8 McGill SM, Kavcic NS, Harvey E. Sitting on a chair or an exercise ball: various perspectives to guide decisions making; examination of the flexion relaxation phenomenon. Clin Biomech (Bristol, Avon). 2006;21:353-60.

9 Van Diëen JH, Looze MP, Hermans V. Effects of dynamic office chair on trunk kinematics, trunk extensor EMG, and spinal shrinkage. Ergonomics. 2001;44(7):739-50.
10 Kigma I, van Diëen JH. Static and dynamic postural loadings during computer work in females: sitting on a chair or an exercise ball. Appl Ergon. 2009;40(2):199205.

11 Collet EN. Background to sitting at work: researchedbased requirement for the design of work seats. Ergonomics. 2006;49(14):1538-46.

12 Carcone SM, Keir PJ. Effects of backrest design on biomechanics and comfort during seated work. Appl Ergon. 2007;38:755-64.

13 Seghers J, Jochem A, Spaepen A. Posture, muscle activity and muscle fatigue in prolonged VDT work at different screen height settings. Ergonomics. 2003;46(7):714-30.

14 Aota Y, lizuka H, Ishigem Y, Mochida T, Yoshihisa T, Uesugi $M$, et al. Effectiveness of a lumbar support continuous passive motion device in the prevention of low-back pain during prolonged sitting. Spine. 2007;32(23):E674-7.

15 Pynt J, Higgs J, Mackey M. Seeking the optimal posture of the seated lumbar spine. Physiother Theory Pract. $2001 ; 17(1): 5-21$.

16 Braccialli LMP, Vilarta R. Aspectos a serem considerados na elaboração de programas de prevenção e orientação de problemas posturais. Rev Paul Educ Fis. 2000;14(2):159-71.

17 Claus AP, Hides JD, Moseley GL, Hodges PW. Is 'ideal' sitting posture real? Measurements of spinal curvatures in four sitting postures. Man Ther. 2009;14(4):404-8. 


\section{Referências (cont.)}

18 Branton P, Grayson G. An evaluation of train seats by observation of sitting behaviour. Ergonomics. $1967 ; 10(1): 35-51$

19 Harrison SS, Harrison SO, Croft AC, Harrison DE, Troyanovich SJ. Sitting biomechanics part I: review of the literature. J Manipulative Physiol Ther. 1999;22(9):594-609.

20 O'Sullivan PB, Dankaerts W, Burnett AF, Farrell GT, Jefford E, Naylor CS, O'Sullivan KJ. Effect of different upright sitting postures on spinal-pelvic curvature and trunk muscle activation in a pain-free population. Spine. 2006;31(19):E707-12.

21 Callaghan JP, Dunk NM. Examination of the flexion relaxation phenomenon in erector spinae muscles during short duration slumped sitting. Clin Biomech (Bristol, Avon). 2002;17(5):353-60.

22 Andersson GBJ, Ortengren R, Nachemson AL, Elfström $\mathrm{G}$, Broman $\mathrm{H}$. The sitting posture: an electromyographic and discometric study. Orthop Clin North Am. 1975;6(1):105-20.

23 Vergara M, Page A. Relationship between comfort and back posture and mobility in sitting posture. Appl Ergon. 2002;33(1):1-8.

24 Adams MA, Hutton WC. The effect of posture on the fluid content of the intervertebral disc. Spine. $1983 ; 8(6): 665-71$.

25 Handa T, Ishihara H, Oshima H, Osada R, Tsuji H, Obata K. Effects of hydrostatic pressure on matrix synthesis and matrix metalloproteinase production in the human lumbar intervertebral disc. Spine. 1997;22(10):1085-91.

26 Oshima H, Tsuji H, Hirano N, Ishihara H, Katoh Y, Yamada $\mathrm{H}$. Water diffusion pathway, swelling pressure, and biomechanical properties of the intervertebral disc during compression load. Spine. 1989;14(11):1234-43.

27 Hedman TP, Fernie GR. Mechanical response of the lumbar spine to seated postural loads. Spine. $1997 ; 22(7): 734-43$

28 Magora A. Investigation of the relation between lowback pain and occupation, part III: physical requirements; sitting, standing, and weight lifting. $\mathrm{Br}$ J Ind Med. 1972;41:5-9.

29 Claus A, Hides J, Moseley GL, Hodges P. Sitting versus standing: does the intradiscal pressure cause disc degeneration or low back pain? J Electromyogr Kinesiol. 2008;18(4):550-8.
30 Nachemson A. The effect of forward leaning on lumbar intradiscal pressure. Acta Orthop Scand. 1965;35:314-28.

31 Wilke HJ, Neef P, Caimi M, Hoogland T, Claes LE. New in vivo measurements of pressures in the intervertebral disc in daily life. Spine. 1999;24(8):755-62.

32 Sato K, Kikuchi S, Yonezawa T. In vivo intradiscal pressure measurement in healthy individuals and in patients ongoing back problems. Spine. 1999; 24(23):2468-72.

33 Gracovetsky S, Kary M, Levy S, Ben SR, Pitchen I, Helie J. Annalysis of spinal and muscular activity during flexion/extension and free lifts. Spine. 1990;15(12):1333-9.

34 O'Sullivan PB, Mitchell T, Bulich P, Waller R, Holte J. The relationship between posture and back muscle endurance in industrial workers with flexion-related low-back pain. Man Ther. 2006;11:264-71.

35 Panjabi MM. The stabilizing system of the spine, part II: neutral zone and stability hipothesis. J Spinal Disord. 1992;5:390-6.

36 Adams MA. Mechanical testing of the spine. Spine. $1995 ; 20: 2151-6$

37 Crisco JJ, Panjabi MM. Euler stability of the human ligamentous lumbar spine, part II: experiment. Clin Biomech (Bristol, Avon). 1992;7(1):27-32.

38 Harrison DD, Harrison SO, Croft AC, Harrison DE, Troyanovich SJ. Sitting biomechanics, part II: optimal car driver's seat and optimal driver's spinal model. J Manipulative Physiol Ther. 2000;23(1):37-47.

39 O'Sullivan PB, Grahamslaw KM, Kendell M, Lapenskie SC, Möller NE, Richards K. The effect of different standing and sitting postures on trunk muscle activity in a pain-free population. Spine. 2002;27(11):1238-44.

40 Fenety PA, Putnam C, Walker JM. In-chair movement: validity, reliability and implications for measuring sitting discomfort. Appl Ergon. 2000;31(4):383-93.

41 Goossens RH, Snidjers CJ, Roelofs JY, van Buchem F. Free shoulder space requirements in the design of high backrests. Ergonomics. 2003;46:518-30.

42 Snidjers CJ, Hermans PFG, Niesing R, Spoors CW, Stoeckart R. The influence of slouching and lumbar support on iliolumbar ligaments, intervertebral discs and sacroiliac joints. Clin Biomech (Bristol, Avon). 2004;19(4):323-9. 
43 Van Deurseir DL, Lengsfeld M, Snidjers CJ, Goossens $\mathrm{RH}$. Mechanical effects of continuous passive motion on the lumbar spine in seating. J Biomech. 2000;33:695-9.

44 Gregory DE, Dunk NM, Callaghan JP. Stability ball versus office chair: comparison of muscle activation and lumbar spine posture during prolonged sitting. Hum Factors. 2006;48(1):142-53.

45 Saarni L, Nygård CH, Kaukiainen A, Rimpelä A. Are the desks and chairs at school appropriate? Ergonomics. 2007;50(10):1561-70.

46 Agha SR. School furniture match to students' anthropometry in the Gaza Strip. Ergonomics. 2010;53(3):344-54.

47 Domljan D, Grbac I, Hadina J. Classroom furniture design: correlation of pupil and chair dimensions. Coll Antropol. 2008;32(1):257-65.

48 Panjabi MM. The stabilizing system of the spine, part I: function, dysfunction, adaptation, and enhancement. J Spinal Disord. 1992;5:383-9.
49 Snidjers CJ, Slagter AHE, van Striker R. Why leg crossing? The influence of common postures on abdominal muscle activity. Spine. 1995;20:1989-93.

50 Gedalia U, Solomonow M, Zhou BH, Baratta RV, Lu Y, Harris M. Biomechanics of increased exposure to lumbar injury caused by cyclic loading, part 2: recovery of reflexive muscular stability with rest. Spine. 1999;24:2461-7.

51 Pelham TW, White $\mathrm{H}$, Holt LE, Lee SW. The etiology of low-back pain in military helicopter aviators: prevention and treatment. Work. 2005;24(2):101-10.

52 Womersley L, May S. Sitting posture of subjects with postural backache. J Manipulative Physiol Ther. 2006;29(3):213-8.

53 Szeto GP, Lam P. Work-related musculoskeletal disorders in urban bus drivers of Hong Kong. J Occup Rehabil. 2007;17:181-98. 